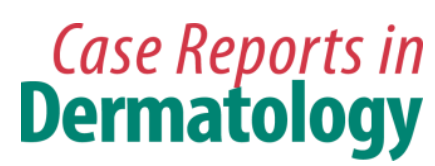

Case Rep Dermatol 2017;9:70-78

DOI: $10.1159 / 000473873$

Publisnea onine: Jury 13, 2017

c) 2017 The Author(s)

Published by S. Karger AG, Basel

www.karger.com/cde

This article is licensed under the Creative Commons Attribution-NonCommercial 4.0 International License (CC BY-NC) (http://www.karger.com/Services/OpenAccessLicense). Usage and distribution for commercial purposes requires written permission.

\title{
A Disease-Modifying Approach for Advanced Hidradenitis Suppurativa (Regimen with Metformin, Liraglutide, Dapsone, and Finasteride): A Case Report
}

\author{
Birgit N. Khandalavala
}

UNMC Department of Family Medicine, Nebraska Medical Center, Omaha, NE, USA

\section{Keywords}

Hidradenitis suppurativa - Acne inversa - Metformin - Liraglutide - Finasteride - Dapsone . Obesity - Weight loss - Metabolic syndrome - Anemia - Leukocytosis - Elevated platelet count . Thrombocytosis · Elevated liver enzymes · Elevated sedimentation rate

\begin{abstract}
Hidradenitis suppurativa (HS) is a challenging skin disease with limited therapeutic options. Obesity and metabolic syndrome are being increasingly implicated and associated with younger ages and greater metabolic severity. A 19-year-old female with an 8-year history of progressively debilitating cicatricial HS disease presented with obesity, profound anemia, leukocytosis, increased platelet count, hypoalbuminemia, and elevated liver enzymes. A combination of metformin, liraglutide, levonorgestrel-ethinyl estradiol, dapsone, and finasteride was initiated. Acute antibiotic use for recurrences and flares could be slowly discontinued. Over the course of 3 years on this regimen, the liver enzymes normalized in 1 year, followed in2 years by complete resolution of the majority of the hematological and metabolic abnormalities. The sedimentation rate reduced from over 120 to $34 \mathrm{~mm} / \mathrm{h}$. She required 1
\end{abstract}

983075 Nebraska Medical Center

Omaha, NE 68198-3075 (USA)

E-Mail birgit.khandalavala@unmc.edu 
surgical intervention for perianal disease after 9 months on the regimen. Flares greatly diminished in intensity and duration, with none in the past 6 months. Right axillary lesions have completely healed with residual disease greatly reduced. Chiefly abdominal lesions are persistent. She was able to complete high school from home, start a job, and resume a normal life. Initial weight loss of 40 pounds was not maintained. The current regimen is being well tolerated and continued.

(C) 2017 The Author(s)

Published by S. Karger AG, Basel

\section{Introduction}

Hidradenitis suppurativa (HS) is a chronic recalcitrant disease of the skin that is typically devastating for the patient. Significantly reduced quality of life with pain commonly ensues in advanced cases [1]. Therapeutic options vary with moderate success, and no current formal guidelines are available [2]. Besides well-known antibiotic regimens, dapsone has been noted to be helpful $[3,4]$. In women, anti-androgen use has been reported to have beneficial outcomes [2]. Recently, finasteride has been found to be highly effective in adults as well as female children, suggesting disease-modifying effects $[5,6]$. While smoking has traditionally been associated with HS [2], obesity and metabolic syndrome are being increasingly implicated and associated with younger incidence and greater metabolic severity [7, 8]. HS disease has been noted to be steadily rising over the last 40 years in the US, ostensibly due to an increase in obesity [9]. Recent studies increasingly indicate a strong association between hidradenitis and obesity [10-18]. Enhanced levels of insulin have been suggested as the linkage due to enhanced bioavailable androgen production [19]. Addressing chronic infection, with a simultaneous reduction of contributing immunometabolic and hormonal factors, would provide a novel therapeutic approach.

Hence, in a young woman with an extensive history of recalcitrant HS and profound medical comorbidities, a unique multimodal regimen was instituted.

\section{Case Report}

A 19-year-old female of South Indian origin with precedent polycystic ovary syndrome (PCOS) and fatty liver presented for evaluation and treatment of obesity and hidradenitis suppurativa in November 2011. Pertinent history was a normal growth and development until puberty with a very active lifestyle. No family history of HS, colitis, or other autoimmune disease was present; however, both maternal grandparents had late-onset diabetes. At the age of 11 years, she immigrated to the United States with her mother and 2 months later begun menstruation. Within 4 months, she had a 45 -pound weight gain. One month later, she developed fever and perianal pain, and underwent surgery for a large perianal abscess. She developed multiple skin lesions consistent with HS. Acne was seen to develop over the face, and periods became irregular. Over the next 6 months, her disease became widespread with up to 20 lesions present at any one time and intermittent perianal disease. Widespread abdominal scarring resulted in contracture of the entire body. She underwent extensive therapy over a total of 8 years with multiple episodic and continuous regimens of oral and intra- 
venous antibiotics, numerous surgeries, isotretinoin for acne treatment, periodic metformin, and oral birth control usage, all without improvement of her disease.

Menstrual periods caused severe flare-ups that required increased pain medication. Sleep was severely impaired.

Physical examination confirmed HS encompassing most of the truncal area anteriorly from the inframammary area to the groin, extending to the right axilla, incorporating nearly $40 \%$ of her body surface, with extensive muscle wasting of the extremities. Facial acne without scarring was present. Hirsutism was notably absent. Her weight was 215 pounds, with a BMI of 37, placing her at class II obesity. Laboratory results are detailed in Table 1 and were notable for profound microcytic anemia with hemoglobin of $6.2 \mathrm{~g} / \mathrm{dL}$ with normal iron indices, and were considered as an anemia of chronic disease along with elevated liver enzymes that were presumed to be due to fatty liver. Her blood sugar was $117 \mathrm{mg} / \mathrm{dL}$ on average due to $\mathrm{HbA}_{1 \mathrm{c}}$ of $5.7 \%$ and qualifies for pre-diabetes.

To offset the weight gain, an aggressive lifestyle modification program was initialed that focused on a low-carbohydrate diet primarily, since physical activity was too painful. Oral contraception was restarted to decrease the flares with menstruation. She was placed on dapsone $100 \mathrm{mg} /$ day, after a glucose-6-phosphate dehydrogenase test was reported as negative. Initial minimal improvements were noted. A few months later, finasteride $5 \mathrm{mg} / \mathrm{day}$ and metformin $2,000 \mathrm{mg} /$ day, followed by liraglutide $0.6 \mathrm{mg}$ subcutaneously daily, increased to $1.8 \mathrm{mg}$ over 2 months, were administered. She lost approximately 40 pounds (19\% body weight) in a 6-month period with her lowest weight recorded at that time, with a trend of regain subsequently. Monthly follow-ups were undertaken with complete laboratory testing at each visit. The liver enzymes responded first with declines within 6 months, and resolution within a year. Leukocytosis, anemia, and hypoalbuminemia returned more gradually to normalcy at 2 years of treatment. The sedimentation rate, though greatly reduced form over 120 to $34 \mathrm{~mm} / \mathrm{h}$, is still above normal, and total proteins remain slightly above normal. $\mathrm{HbA}_{1 \mathrm{c}}$ was $5.2 \%$ after 2 years and $4.9 \%$ after 3 years with average estimated blood sugar of $94 \mathrm{mg} / \mathrm{d}$; though weight was regained (Fig. 2). Blood testing was reduced to every 3 months after 2 years of the regimen since the improvements were stable and maintained.

The clinical course did not respond initially as well as the laboratory findings, with no improvement in the first 3 months. Then, new lesions did appear but resolved faster. The patient began to feel better and did have less intense and frequent flares about 6 months into the regimen. Nine months later, another large perianal abscess developed that required hospitalization and surgery. The perianal lesion healed completely in 3 weeks, as compared to a similar instance 3 years prior, which needed 6 months of dressings and care. The patient elected to avoid any extensive debridement. In the past year, she has not required adjunctive antibiotics, and risk of pregnancy, though addressed, has not been a concern.

At present, she has not had a new lesion for 6 months and has completed 3 years of the regimen, with remarkable tolerance. Axillary lesions have healed completely, groin, thigh, and perianal disease have improved $90 \%$ or more, while thoraco-abdominal lesions have improved $60 \%$ with 4-5 small areas persisting. Facial acne has improved but persisted. During the frequent follow-up visits, adverse effects were asked for routinely and weight regain was noted. No physical or biochemical abnormality was detected, and only the sedimentation rate is minimally elevated. The patient did consent to having photographs of the right 
axilla only: Figure 1, Figure 2, and Figure 3 show the current condition and complete healing of the right axilla. Previous photographs were not obtained due to the contracture.

\section{Discussion}

HS is a particularly challenging disease for the clinician, with disease modification seldom achieved. Medical co-morbidities contribute to the desperate situation and complexity. With the growing importance of metabolic syndrome and the concept that HS is a disease primarily of immunity with concomitant infection, therapy that targets the local and systemic hormonal and immunometabolic aspects would seem a prudent, though unknown approach. Review of the current literature (PubMed, EMBASE, Cochrane database) did not reveal any other reports of the use of finasteride with dapsone, or combinations with metformin and liraglutide. I report for the first time remission of a case of HS and nearly all associated medical co-morbidities with a multimodal approach that could be considered curative. Dapsone has been used for HS with mostly favorable outcomes and tolerance, with few side effects [3, 4]. Dapsone combines both bacteriostatic as well as immunological effects [3].

Hypersensitivity is known [20] and I did opt to rule out glucose-6-phosphate dehydrogenase, due to the anemia. Finasteride has been used in HS [5] with excellent outcomes. Most recently, the medication has been found to be highly beneficial in female pediatric aged patients, used over a period of 6 years with no significant adverse effects [6]. Finasteride is thought to alter the androgen sensitivity of the hair follicle and impact the local action of the bioactive dihydrotestosterone (DHT) rather than reducing biochemical hyperandrogenism [5]. Teratogenicity in females must be addressed and contraception advocated in all women of reproductive age. For this patient, oral birth control was instituted for the premenstrual flares, but acne has continued to be an issue.

Metformin is an anti-hyperglycemic agent that promotes insulin sensitivity [21]. It is used extensively across the world, for diabetes, pre-diabetes, and PCOS, and has well-known gastrointestinal adverse effects but is relatively safe. My patient had both pre-diabetes and PCOS. In such patients, metformin reduces insulin resistance and insulin levels, which lowers serum androgen concentrations. Metformin, when administered to lean, overweight, and moderately obese women with PCOS, reduces serum luteinizing hormone and increases follicle stimulating hormone and sex hormone-binding globulin. Up to 50\% reduction in serum testosterone concentrations may be detected [13]. Metformin has recently been found to control HS with minimal side effects [19].

Liraglutide is an incretin mimetic glucagon-like peptide -1 receptor agonist currently indicated for type 2 diabetes mellitus [14] and has been approved by the FDA for a weight loss indication [22]. Both insulin synthesis and secretion from the pancreatic beta cells is stimulated in the presence of elevated glucose, while suppressing glucagon secretion, slowing gastric emptying, with reduction in food intake [22]. Both metformin and liraglutide were well tolerated and symptoms suggestive of hypoglycemia were absent. In spite of improvement in the metabolic profile and initial weight loss, weight regain has been ongoing. In their study, Revuz et al. [23] concluded that obesity was strongly correlated with the degree of HS and suggested a causal role. My case would seem to infer that obesity is not correlated as 
long as the metabolic parameters are controlled as evident by clinical remission in spite of weight regain.

Certainly, using a combination does not allow for evaluation of the individual components, and step therapy could be a future option with enhanced immunometabolic and hormonal testing that could include insulin levels and other specific hormones. Monthly followup and evaluations were conducted for 2 years, to clearly confirm regression and closely monitor for the onset of other adverse effect, though reassuring ideal timing is unknown.

There did appear to be latency to the onset of improvement, and the current conundrum remains as to withdrawing one of the components since this regimen use is unprecedented and relapse has been noted with discontinuation of dapsone or finasteride when used as mono-therapy. With the long-term data of finasteride showing excellent outcomes after continuous use of 6 years, long-term use would appear to be indicated. Dapsone has been used long term for other skin disorders, and both metformin and liraglutide are indicated for long term. While the vast majority of her initial hematological and metabolic improvement is nearly complete, the sedimentation rate may continue to remain elevated due to persistent abdominal lesions. If weight gain is persistent, a newer formulation of liraglutide could be used when available [22].

This case study provokes more discussion and advocates combination therapies to be implemented more intensively than the current norm, especially anticipating the rising global epidemic of obesity and metabolic syndrome.

In summary, for a disease as complex and overwhelming as HS, a multimodal approach targeting the metabolic and immunohormonal aspects predominantly, has resulted in the case of a young lady with obesity to have a disease-modifying effect, with resolution of all major concomitant medical abnormalities.

\section{Statement of Ethics}

The patient did consent to having photographs of the right axilla.

\section{Disclosure Statement}

Dr. Birgit Khandalavala has received no financial support for this article and has no conflicts of interest.

\section{References}

1 Scheinfeld N: Hidradenitis suppurativa: a practical review of possible medical treatments based on over 350 hidradenitis patients. Dermatol Online J 2013;19:1.

2 Jemec GBE: Hidradenitis suppurativa. N Engl J Med 2012;366:158-164.

-3 Yazdanyar S, Boer J, Ingvarsson G, Szepietowski JC, Jemec GBE: Dapsone therapy for hidradenitis suppurativa: a series of 24 patients. Dermatology 2011;222:342-346.

4 Kaur MR, Lewis HM: Hidradenitis suppurativa treated with dapsone: a case series of five patients. J Dermatolog Treat 2006;17:211-213. 
5 Joseph MA, Jayaseelan E, Ganapathi B, Stephen J: Hidradenitis suppurativa treated with finasteride. J Dermatolog Treat 2005;16:75-78.

6 Randhawa HK, Hamilton J, Pope E: Finasteride for the treatment of hidradenitis suppurativa in children and adolescents. JAMA Dermatol 2013;149:732-735.

7 Sabat R, Chanwangpong A, Schneider-Burrus S, Metternich D, Kokolakis G, et al: Increased prevalence of metabolic syndrome in patients with acne inversa. PLoS One 2012;7:e31810.

-8 Gold DA, Reeder VJ, Mahan MG, Hamzavi IH: The prevalence of metabolic syndrome in patients with hidradenitis suppurativa. J Am Acad Dermatol 2014;70:699-703.

-9 Vazquez BG, Alikhan A, Weaver AL, Wetter DA, Davis MD: Incidence of hidradenitis suppurativa and associated factors: a population-based study of Olmsted County, Minnesota. J Invest Dermatol 2013;133:97-103.

10 Silva-Feistner M, Salomone C, Silva S, Vial-Letelier V: Prepubertal hidradenitis suppurativa: report of 2 clinical cases. Rev Chil Pediatr 2016;87:193-198.

11 Kohorst JJ, Kimball AB, Davis MD: Systemic associations of hidradenitis suppurativa. J Am Acad Dermatol 2015;73(5 suppl 1):S27-S35.

12 Schrader AM, Deckers IE, van der Zee HH, Boer J, Prens EP: Hidradenitis suppurativa: a retrospective study of 846 Dutch patients to identify factors associated with disease severity. J Am Acad Dermatol 2014;71:460-467.

13 Kromann CB, Deckers IE, Esmann S, Boer J, Prens EP, Jemec GB: Risk factors, clinical course and longterm prognosis in hidradenitis suppurativa: a cross-sectional study. Br J Dermatol 2014;171:819-824.

14 Kromann CB, Ibler KS, Kristiansen VB, Jemec GB: The influence of body weight on the prevalence and severity of hidradenitis suppurativa. Acta Derm Venereol 2014;94:553-557.

15 Gold DA, Reeder VJ, Mahan MG, Hamzavi IH: The prevalence of metabolic syndrome in patients with hidradenitis suppurativa. J Am Acad Dermatol 2014;70:699-703.

-16 Canoui-Poitrine F, Le Thuaut A, Revuz JE, Viallette C, Gabison G, Poli F, Pouget F, Wolkenstein P, BastujiGarin S: Identification of three hidradenitis suppurativa phenotypes: latent class analysis of a crosssectional study. J Invest Dermatol 2013;133:1506-1511.

17 Sabat R, Chanwangpong A, Schneider-Burrus S, Metternich D, Kokolakis G, Kurek A, Philipp S, Uribe D, Wolk K, Sterry W: Increased prevalence of metabolic syndrome in patients with acne inversa. PLoS One 2012;7:e31810.

18 Sartorius K, Emtestam L, Jemec GB, Lapins J: Objective scoring of hidradenitis suppurativa reflecting the role of tobacco smoking and obesity. Br J Dermatol 2009;161:831-839.

$>19$ Verdolini R: Metformin for the treatment of hidradenitis suppurativa: a little help along the way. J Eur Acad Dermatol Venereol 2013;27:1101-1108.

20 Wozel G: Dapsone in dermatology and beyond. Arch Dermatol Res 2014;306:103-124.

21 Metformin. http://www.clinicalpharmacologyip.com/Forms/Monograph/monograph.aspx?cpnum=379\&sec=mondesc\&t=0. Published 2014. Updated 2014. Accessed November 17, 2014.

22 Liraglutide. http://www.clinicalpharmacologyip.com/Forms/Monograph/monograph.aspx?cpnum=3496\&sec=monmech\&t=0. Published 2014. Updated 2012. Accessed November, 17, 2014.

23 Revuz JE, Canoui-Poitrine F, Wolkenstein P, et al: Prevalence and factors associated with hidradenitis suppurativa: results from two case-control studies. J Am Acad Dermatol 2008;59:596-601.

Dr. Khandalavala is Associate Professor of family medicine and board certified in obesity medicine at the University of Nebraska Medical Center. 


\section{Case Reports in Dermatology}

\begin{tabular}{l|l}
\hline DOI: $10.1159 / 000473873$ & C 2017 The Author(s). Published by S. Karger AG, Basel
\end{tabular} www.karger.com/cde

Khandalavala: A Disease-Modifying Approach for Advanced Hidradenitis Suppurativa

(Regimen with Metformin, Liraglutide, Dapsone, and Finasteride): A Case Report

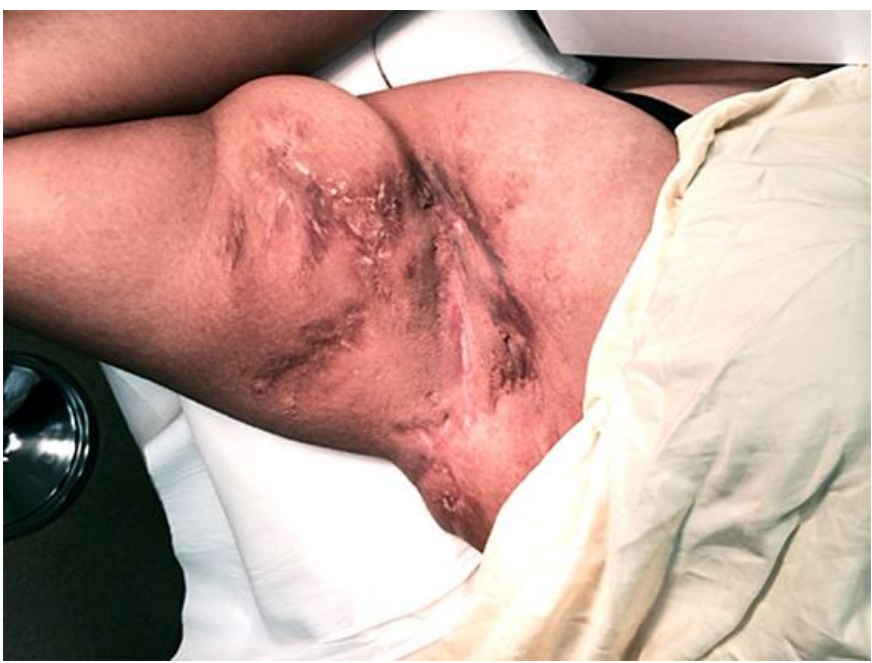

Fig. 1. Hurley stage III hidradentitis suppurativa of the right axilla in remission following 36 months of treatment (view 1). 


\section{Case Reports in Dermatology}

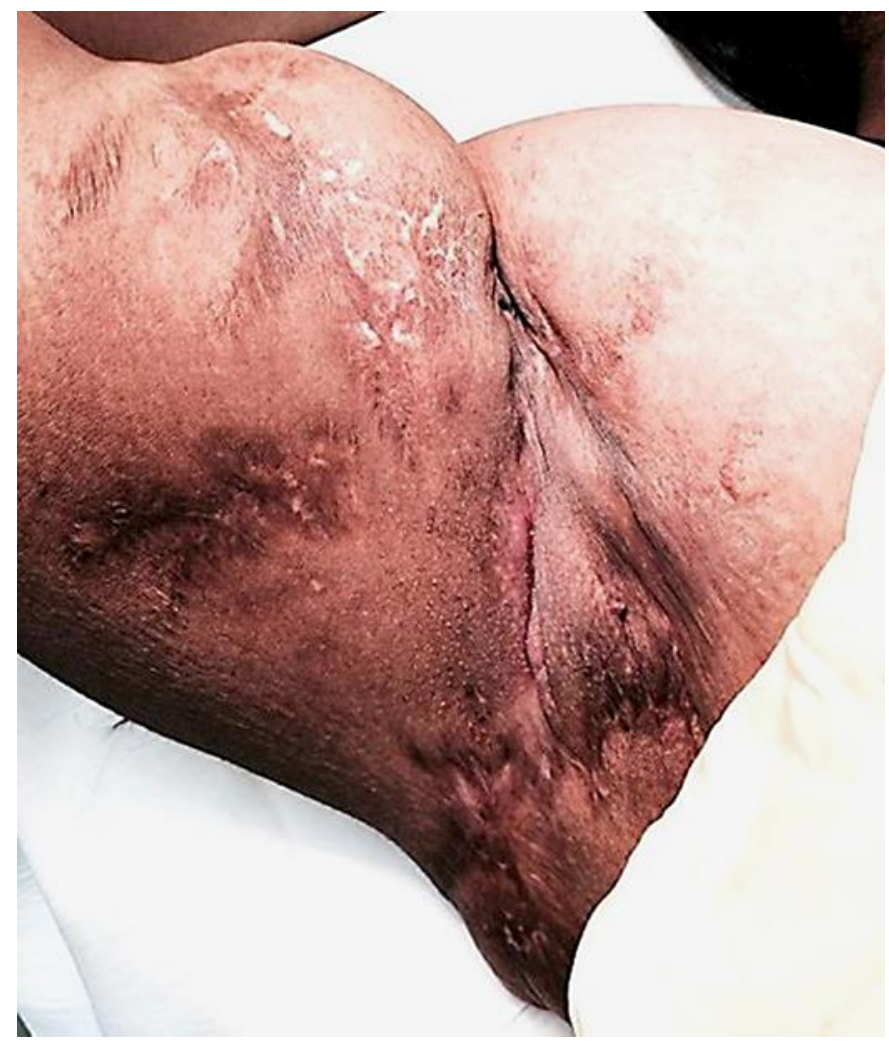

Fig. 2. Hurley stage III hidradentitis suppurativa of the right axilla in remission following 36 months of treatment (view 2).

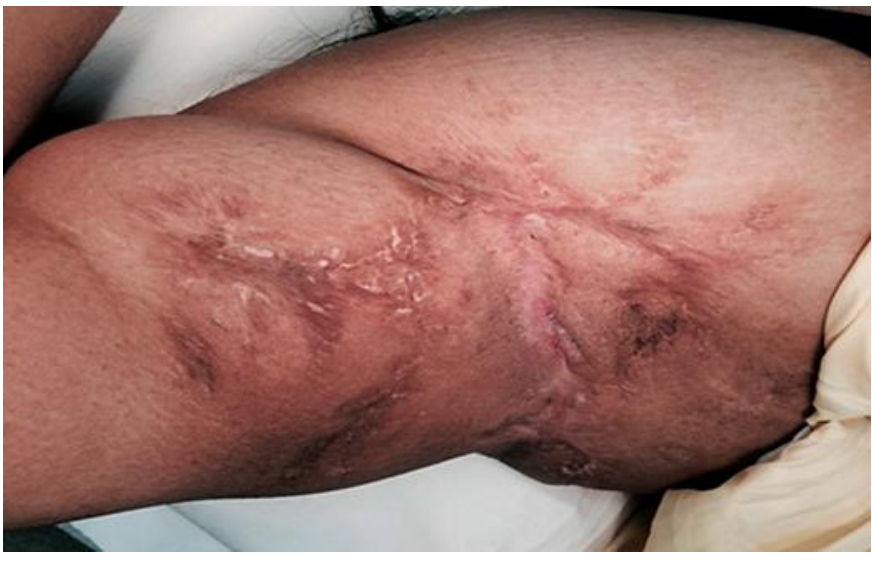

Fig. 3. Hurley stage III hidradentitis suppurativa of the right axilla in remission following 36 months of treatment (view 3). 
Table 1. Laboratory results

\begin{tabular}{llllll}
\hline & Baseline & 6 months & 1 year & 2 years & 3 years \\
\hline WBC, / $\mu \mathrm{L}$ & $\mathbf{3 4 \times \mathbf { 1 0 } ^ { 3 }}$ & $\mathbf{3 2 \times \mathbf { 1 0 } ^ { 3 }}$ & $\mathbf{1 8 . 8 \times \mathbf { 1 0 } ^ { 3 }}$ & $9.7 \times 10^{3}$ & $9.2 \times 10^{3}$ \\
Hemoglobin, g/dL & $\mathbf{6 . 2}$ & $\mathbf{8 . 3}$ & $\mathbf{8 . 5}$ & 11.8 & 12.5 \\
Platelet, $/ \mu \mathrm{L}$ & $\mathbf{7 8 3 \times \mathbf { 1 0 } ^ { 3 }}$ & $\mathbf{5 3 5 \times \mathbf { 1 0 } ^ { 3 }}$ & $\mathbf{5 2 3 \times \mathbf { 1 0 } ^ { 3 }}$ & $376 \times 10^{3}$ & $391 \times 10^{3}$ \\
Sedimentation rate, mm/h & $\mathbf{1 1 2 0}$ & $\mathbf{1 1 2 0}$ & $\mathbf{1 0 9}$ & $\mathbf{7 4}$ & $\mathbf{3 4}$ \\
Glucose, mg/dL & $\mathbf{1 3 0}$ & 105 & 87 & 98 & 89 \\
AST, U/L & 55 & $\mathbf{2 7}$ & 19 & 26 & 24 \\
ALT, U/L & 112 & $\mathbf{7 2}$ & $\mathbf{5 7}$ & 34 & 31 \\
Albumin, g/dL & $\mathbf{3 . 2}$ & $\mathbf{3 . 2}$ & 4.0 & 3.8 & 4.1 \\
HbA1c, \% & $\mathbf{5 . 7}$ & 5.2 & 5.2 & 5.0 & 4.9 \\
\hline
\end{tabular}

Bolded numbers represent abnormal laboratory values. 